Article

\title{
A VaR-Type Risk Measure Derived from Cumulative Parisian Ruin for the Classical Risk Model
}

\author{
Mohamed Amine Lkabous *,† and Jean-François Renaud ${ }^{\dagger}$ \\ Département de mathématiques, Université du Québec à Montréal (UQAM), Montréal, QC H2X 3Y7, Canada; \\ renaud.jf@uqam.ca \\ * Correspondence: lkabous.mohamed_amine@courrier.uqam.ca; Tel.: +1-514-987-4186 \\ † These authors contributed equally to this work.
}

Received: 30 July 2018; Accepted: 23 August 2018; Published: 24 August 2018

\begin{abstract}
In this short paper, we study a VaR-type risk measure introduced by Guérin and Renaud and which is based on cumulative Parisian ruin. We derive some properties of this risk measure and we compare it to the risk measures of Trufin et al. and Loisel and Trufin.
\end{abstract}

Keywords: risk measure; cumulative Parisian ruin; stochastic orders; surplus process

\section{Introduction}

Over the last few years, several dynamic risk measures, i.e., risk measures based on ruin-theoretic quantities, have been studied. For example, in the classical compound Poisson risk model, Trufin et al. (2011) considered a VaR-type risk measure defined as the smallest initial capital needed to ensure a certain probability of solvency throughout the lifetime of the surplus process. This risk measure has been extended by Mitric and Trufin (2016) who defined a risk measure taking into account both the probability of ruin and the expected deficit at ruin. In addition, Loisel and Trufin (2014) used the expected area below the solvency threshold as a risk indicator to introduce a new risk measure with some interesting properties.

Very recently, implementation delays in the recognition of ruin and occupation times of the surplus process have been used as alternative risk management tools to assess the quality of an insurance portfolio. In this direction, Guérin and Renaud (2017) introduced the concept of cumulative Parisian ruin, which is based on the time spent in the red by the underlying surplus process. The time of cumulative Parisian ruin is the first time the surplus process stays cumulatively below a critical level longer than a pre-determined grace period. Inspired by the risk measure of Trufin et al. (2011), they defined a VaR-type risk measure based on cumulative Parisian ruin. It is also defined as the smallest amount of capital for which the associated cumulative Parisian ruin probability is less than or equal to a tolerable level.

In this paper, we study this VaR-type risk measure based on cumulative Parisian ruin. In Guérin and Renaud (2017), this risk measure is proposed as a motivational reason to study the concept of cumulative Parisian ruin; the risk measure itself is neither analyzed nor used for any particular application. We derive some of its properties and compare it to the risk measures of Trufin et al. (2011) and Loisel and Trufin (2014).

The rest of the paper is organized as follows. In Section 2, we recall some background on the Cramér-Lundberg model, also known as the classical risk model, and define the concept of cumulative Parisian ruin. In Section 3, we introduce our risk measure and we give some of its properties. Finally, in Section 4, we study our risk measure in the special case of a Cramér-Lundberg process with exponential claims. 


\section{Insurance Risk Model}

The Cramér-Lundberg model was proposed by Lundberg (1903) and further developed by Cramér (1930). In this model, the surplus process of an insurance company is modelled by

$$
X_{t}=x+c t-S_{t}
$$

where $x \geq 0$ and $c>0$, and where $S_{t}=\sum_{i=1}^{N_{t}} C_{i}$ is a compound Poisson process with $N=\left\{N_{t}, t \geq 0\right\}$ a Poisson process of intensity $\lambda>0$ and with $\left\{C_{1}, C_{2}, \ldots\right\}$ positive random variables following a common cumulative distribution function $F_{C}$. Recall that in this setup the claim sizes $\left\{C_{1}, C_{2}, \ldots\right\}$ are mutually independent and are also independent of the number-of-claim process $N$. The process $S=\left\{S_{t}, t \geq 0\right\}$ is known as the aggregate claim amount process. We call $x$ the initial capital and $c$ the premium rate.

We use the following equivalent notations $\mathbb{P}_{x}(\cdot) \equiv \mathbb{P}\left(\cdot \mid X_{0}=x\right)$ to emphasize that the process $X$ starts at level $x$. The notation $\mathbb{E}_{x}$ corresponds to $\mathbb{P}_{x}$. When $X_{0}=0$, we drop the index. In this model, the premium rate $c$ is chosen usually to satisfy the net profit condition $\mathbb{E}\left[X_{1}\right]=c-\lambda \mathbb{E}\left[C_{1}\right]>0$, which means that we can define the safety loading factor $\eta>0$ by $\eta:=\left(c-\lambda \mathbb{E}\left[C_{1}\right]\right) / \lambda \mathbb{E}\left[C_{1}\right]$.

The time of classical ruin associated to $X$ is defined as

$$
\tau_{0}^{-}=\inf \left\{t>0: X_{t}<0\right\}
$$

We denote the corresponding finite-time probability of ruin, for $x \geq 0$ and $t>0$, by

$$
\psi(t, x)=\mathbb{P}_{x}\left(\tau_{0}^{-} \leq t\right)
$$

and the infinite-time probability of ruin by

$$
\psi(x)=\mathbb{P}_{x}\left(\tau_{0}^{-}<\infty\right) .
$$

Of course, we have $\psi(x)=\lim _{t \rightarrow \infty} \psi(t, x)$.

In Trufin et al. (2011), assuming that the safety loading $\eta$ is fixed, the following ruin-consistent VaR-type risk measure is defined and analyzed: for a tolerance level $\epsilon>0$, set

$$
\zeta_{\epsilon}[C]=\inf \{x \geq 0: \psi(x) \leq \epsilon\}
$$

It is well known that we can compute $\psi(x)$ using the Pollaczeck-Khinchine formula (also known in the actuarial literature as the Beekman's convolution formula, see Beekman (1985)) which states that the probability of classical ruin is equal to the tail distribution function of a compound geometric random variable. First, let us define the aggregate loss at time $t$ by $L_{t}=S_{t}-c t$ and the maximal aggregate loss of the process by $L=\max _{t \geq 0} L_{t}$. The random variable $L$ can be expressed as

$$
L=\sum_{i=1}^{M} D_{i}
$$

where $M$ is the number of record highs, which has a geometric distribution with success probability $\eta /(\eta+1)$, and where $\left\{D_{1}, D_{2}, \ldots\right\}$ are the ladder heights with common distribution $F_{D}(u)=\int_{0}^{u}\left(1-F_{C}(y)\right) \mathrm{d} y / \mathbb{E}\left[C_{1}\right]$. The Pollaczeck-Khinchine formula for the probability of ruin is then given by

$$
\psi(x)=\mathbb{P}(L>x)=1-\frac{\eta}{\eta+1} \sum_{k=1}^{\infty}\left(\frac{1}{\eta+1}\right)^{k} F_{D}^{*(k)}(x)
$$


where $F_{D}^{*(k)}$ denotes the $k$-th convolution of the distribution $F_{D}$. Therefore, this risk measure can also be written as follows:

$$
\zeta_{\epsilon}[C]=\inf \{x \geq 0: \mathbb{P}(L>x) \leq \epsilon\}=F_{L}^{-1}(1-\epsilon) .
$$

In some sense, the focus of this risk measure is shifted from the surplus process $X$ to the distribution of the maximal aggregate loss $L$. This important relationship is at the core of the analysis done in Trufin et al. (2011). However, this relationship with the maximal aggregate loss $L$ does not exist for the finite-time ruin probability. Note that this is also the case for the VaR-type risk measure defined and analyzed in Mitric and Trufin (2016).

\section{Cumulative Parisian Ruin}

Recently, Guérin and Renaud (2017) introduced a new definition of actuarial ruin based on the occupation-time process (below 0 ) associated with the surplus process $X$. The occupation-time process $\mathcal{O}^{L}=\left\{\mathcal{O}_{t}^{L}, t \geq 0\right\}$ is defined as

$$
\mathcal{O}_{t}^{L}=\int_{0}^{t} \mathbf{1}_{\left\{X_{u}<0\right\}} \mathrm{d} u=\int_{0}^{t} \mathbf{1}_{\left\{L_{u}>X_{0}\right\}} \mathrm{d} u .
$$

Then, the time of cumulative Parisian ruin, with delay $r>0$, is given by

$$
\sigma_{r}=\inf \left\{t>0: \mathcal{O}_{t}^{L}>r\right\} .
$$

In the definition of cumulative Parisian ruin, we aggregate the duration of all periods of financial distress until we accumulate $r$ units of time spent in that red zone. Consequently, ruin is not declared as soon as $X$ goes below zero: for $x \geq 0, t>0$ and $r>0$, we have

$$
\mathbb{P}_{x}\left(\sigma_{r} \leq t\right) \leq \mathbb{P}_{x}\left(\tau_{0}^{-} \leq t\right)
$$

Cumulative Parisian ruin is somehow a generalization of classical ruin and, when $r \rightarrow 0$, we recover the classical definition (see Guérin and Renaud (2017) for the details and see Figure 1 for a graphical comparison).

We denote the finite-time probability of cumulative Parisian ruin by

$$
\psi_{r}(t, x)=\mathbb{P}_{x}\left(\sigma_{r} \leq t\right)=\mathbb{P}_{x}\left(\mathcal{O}_{t}^{L}>r\right)
$$

and the infinite-time version by

$$
\psi_{r}(x)=\mathbb{P}_{x}\left(\sigma_{r}<\infty\right) .
$$

Of course, we have $\psi_{r}(x)=\lim _{t \rightarrow \infty} \psi_{r}(t, x)$. With this new notation in hand, we can re-write the inequality in Equation (7) as follows: for $x \geq 0, t>0$ and $r>0$, we have

$$
\psi_{r}(t, x) \leq \psi(t, x) .
$$

We also have

$$
\psi(t, x)=\lim _{r \rightarrow 0} \psi_{r}(t, x) \quad \text { and } \quad \psi(x)=\lim _{r \rightarrow 0} \psi_{r}(x) .
$$




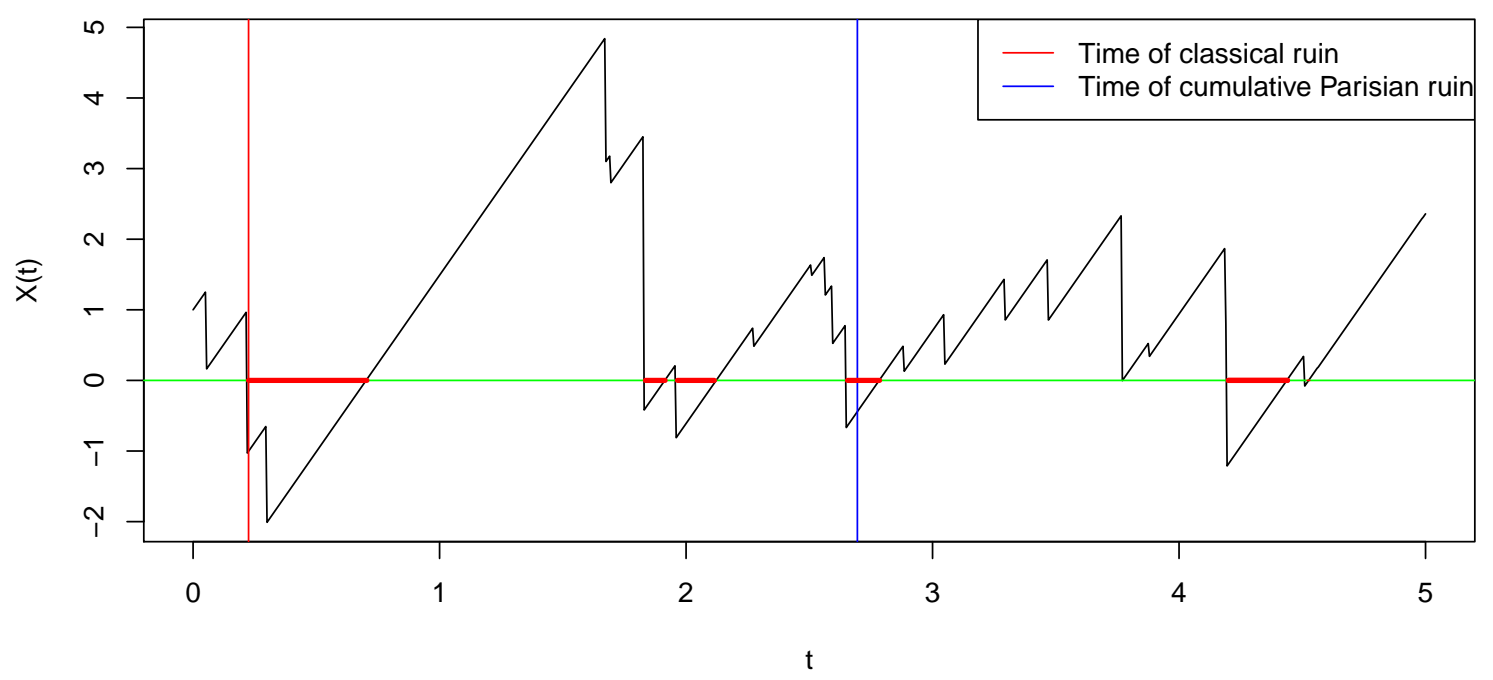

Figure 1. A sample path of a Cramér-Lundberg process $X_{t}$. The time of ruin $\tau_{0}^{-}$is in red and the time of cumulative Parisian ruin $\kappa_{r}$ is in blue.

\section{A VaR-type Risk Measure Derived from Cumulative Parisian Ruin}

Using the definition of cumulative Parisian ruin, Guérin and Renaud (2017) defined the following VaR-type risk measure: for a time horizon of length $t$ and delay $r$, and for a given tolerance level $\epsilon>0$, set

$$
\rho_{\epsilon}^{(r, t)}[X]=\inf \left\{x \geq 0: \psi_{r}(t, x) \leq \epsilon\right\} .
$$

It gives the amount of initial capital needed in order to bound the finite-time probability of cumulative Parisian ruin with delay $r$ by $\epsilon$. Since $\psi_{r}(t, x)=\mathbb{P}_{x}\left(\mathcal{O}_{t}^{L}>r\right)$, we can also write

$$
\rho_{\epsilon}^{(r, t)}[L]=\inf \left\{x \geq 0: \mathbb{P}_{x}\left(\mathcal{O}_{t}^{L}>r\right) \leq \epsilon\right\} .
$$

Consequently, this risk measure is based on the distribution of $\mathcal{O}_{t}^{L}$. This is the analog of the random variable $L$ for the risk measure in Equation (6). A major improvement is that we can now vary both the time horizon and the implementation delay by changing the values of $t$ and $r$, respectively. The trade-off is that we need the distribution of a strongly path-dependent random variable, namely $\mathcal{O}_{t}^{L}$.

For the rest of this paper, we focus on the properties of this VaR-type cumulative Parisian risk measure. In addition, we compare the infinite-time version to the infinite-time risk measure defined in Trufin et al. (2011). Then, we also study the finite-time version as this is possible as soon as the distribution of $\mathcal{O}_{t}^{L}$ is available.

Before going any further, let us give some background material on stochastic dominance.

\subsection{Stochastic Dominance}

Consider two random variables $X$ and $Y$, and let $\bar{F}_{X}$ and $\bar{F}_{Y}$ be their survival functions. We say that $X$ is smaller than $Y$ in the stochastic dominance order, which is denoted by $X \preceq_{s t} Y$, if

$$
\bar{F}_{X}(u) \leq \bar{F}_{Y}(u) \text {, for all } u .
$$

Equivalently, for all non-decreasing functions $\phi$, we have

$$
\mathbb{E}[\phi(X)] \leq \mathbb{E}[\phi(Y)] .
$$

Here is a theorem taken from Shaked and Shanthikumar (2007). 


\section{Theorem 1.}

(i) Let $\left\{X_{1}, X_{2}, \ldots, X_{m}\right\}$ and $\left\{Y_{1}, Y_{2}, \ldots, Y_{m}\right\}$ be two finite sets of independent random variables such that $X_{i} \preceq_{s t} Y_{i}$, for each $i=1, \ldots, m$. Then, for any increasing function $g: \mathbb{R}^{m} \rightarrow \mathbb{R}$, we have

$$
g\left(X_{1}, X_{2}, \ldots, X_{m}\right) \preceq_{s t} g\left(Y_{1}, Y_{2}, \ldots, Y_{m}\right)
$$

(ii) Consider two sequences of random variables $\left\{X_{1}, X_{2}, \ldots\right\}$ and $\left\{Y_{1}, Y_{2}, \ldots\right\}$ and two random variables $X$ and $Y$ such that

$$
X_{n} \stackrel{d}{\rightarrow} X \text { and } Y_{n} \stackrel{d}{\rightarrow} Y
$$

where $\stackrel{d}{\rightarrow}$ denotes convergence in distribution. If $X_{n} \preceq_{s t} Y_{n}$ for each $n$, then $X \preceq_{s t} Y$.

(iii) Let the positive integer-valued random variable $N$ be independent of the family of random variables $\left\{C_{1}, C_{2}, \ldots\right\}$ and define $S=\sum_{i=1}^{N} C_{i}$. Define similarly $\tilde{S}=\sum_{i=1}^{\tilde{N}} \tilde{C}_{i}$.

If $N \preceq_{s t} \tilde{N}$ and $C_{i} \preceq_{s t} \tilde{C}_{i}$ for each $i$, then

$$
S \preceq_{s t} \tilde{S} .
$$

Finally, if $X=\left\{X_{t}, t \geq 0\right\}$ and $Y=\left\{Y_{t}, t \geq 0\right\}$ are stochastic processes, then we write $X \preceq_{s t} Y$ if, for each $t \geq 0$, we have

$$
X_{t} \preceq s t Y_{t} .
$$

The reader is referred to Shaked and Shanthikumar (2007), Kaas et al. (2008) and Denuit et al. (2005) for more details on stochastic ordering and applications in actuarial science.

3.2. Properties of the Risk Measure $\rho_{\epsilon}^{(r, t)}$

In the following, let $L$ and $\tilde{L}$ be two aggregate loss amount processes associated with two aggregate claim amount processes $S$ and $\tilde{S}$, themselves from two Cramér-Lundberg risk processes $X$ and $\tilde{X}$ as defined in $\mathrm{v}(1)$.

Theorem 2. For $r>0, \varepsilon>0$ and $t>0$, we have:

(i) Invariance by translation: For $a>0$,

$$
\rho_{\epsilon}^{(r, t)}[L+a]=\rho_{\epsilon}^{(r, t)}[L]-a .
$$

(ii) Positive homogeneity: For $b>0$,

$$
\rho_{\epsilon}^{(r, t)}[b L]=b \rho_{\epsilon}^{(r, t)}[L] .
$$

(iii) Monotonicity: If $L \preceq_{s t} \tilde{L}$, then

$$
\rho_{\epsilon}^{(r, t)}[L] \leq \rho_{\epsilon}^{(r, t)}[\tilde{L}]
$$

Proof. First, note that

$$
\mathbb{P}_{x}\left(\mathcal{O}_{t}^{L+a}>r\right)=\mathbb{P}_{x}\left(\int_{0}^{t} \mathbf{1}_{\left\{L_{u}>x-a\right\}} \mathrm{d} u>r\right)=\mathbb{P}_{x-a}\left(\mathcal{O}_{t}^{L}>r\right) .
$$


Consequently,

$$
\begin{aligned}
\rho_{\epsilon}^{(r, t)}[L+a] & =\inf \left\{x \geq 0: \mathbb{P}_{x}\left(\mathcal{O}_{t}^{L+a}>r\right) \geq \epsilon\right\} \\
& =\inf \left\{x \geq 0: \mathbb{P}_{x+a}\left(\mathcal{O}_{t}^{L}>r\right) \geq \epsilon\right\} \\
& =\rho_{\epsilon}^{(r, t)}[L]+a .
\end{aligned}
$$

This proves Equation (14).

Similarly, if we note that

$$
\mathbb{P}_{x}\left(\mathcal{O}_{t}^{b L}>r\right)=\mathbb{P}_{x}\left(\int_{0}^{t} \mathbf{1}_{\left\{L_{u}>x / b\right\}} \mathrm{d} u>r\right)=\mathbb{P}_{x / b}\left(\mathcal{O}_{t}^{L}>r\right),
$$

then Equation (15) follows.

To prove the third property, we fix $t>0$ and we show that, if $L_{u} \preceq_{s t} \tilde{L}_{u}$ for all $u \leq t$, then

$$
\mathcal{O}_{t}^{L} \preceq_{s t} \mathcal{O}_{t}^{\tilde{L}} .
$$

First, let us define a sequence of discretized versions of the occupation-time process $\mathcal{O}_{t}^{L}$. For each $n \geq 1$, choose $0=t_{0}<t_{1}<\ldots<t_{n}=t$ such that $\max _{0 \leq i \leq n}\left(t_{i}-t_{i-1}\right) \rightarrow 0$, as $n \rightarrow \infty$, and define

$$
\mathcal{O}_{t}^{(n)}=\sum_{i=1}^{n}\left(t_{i}-t_{i-1}\right) \mathbf{1}_{\left\{L_{t_{i}}>x\right\}} \text {. }
$$

We define $\widetilde{\mathcal{O}}_{t}^{(n)}$ in the obvious way, i.e., when $S$ is replaced by $\tilde{S}$. We can re-write $\mathcal{O}_{t}^{(n)}$ as follows:

$$
\mathcal{O}_{t}^{(n)}=\phi_{n}\left(L_{t_{1}}-L_{t_{0}}, L_{t_{2}}-L_{t_{1}}, \ldots, L_{t_{n}}-L_{t_{n-1}}\right),
$$

where $\phi_{n}\left(u_{1}, \ldots, u_{n}\right)=\sum_{i=1}^{n}\left(t_{i}-t_{i-1}\right) \mathbf{1}_{\left\{\sum_{j=1}^{i} u_{j}>x+c t_{i}\right\}}$.

Since $L_{u} \preceq_{s t} \tilde{L}_{u}$ for all $u \leq t$, then we have $L_{t_{i}-t_{i-1}} \preceq_{s t} \tilde{L}_{t_{i}-t_{i-1}}$ for each $i$. Then, since

$$
L_{t_{i}-t_{i-1}} \stackrel{d}{=} L_{t_{i}}-L_{t_{i-1}} \quad \text { and } \quad \tilde{L}_{t_{i}-t_{i-1}} \stackrel{d}{=} \tilde{L}_{t_{i}}-\tilde{L}_{t_{i-1}}
$$

we have that $L_{t_{i}}-L_{t_{i-1}} \preceq_{s t} \tilde{L}_{t_{i}}-\tilde{L}_{t_{i-1}}$ for each $i$. From Equation (12), we obtain

$$
\phi_{n}\left(L_{t_{1}}-L_{t_{0}}, L_{t_{2}}-L_{t_{1}}, \ldots, L_{t_{n}}-L_{t_{n-1}}\right) \preceq_{s t} \phi_{n}\left(\tilde{L}_{t_{1}}-\tilde{L}_{t_{0}}, \tilde{L}_{t_{2}}-\tilde{L}_{t_{1}}, \ldots, \tilde{L}_{t_{n}}-\tilde{L}_{t_{n-1}}\right),
$$

or equivalently

$$
\mathcal{O}_{t}^{(n)} \preceq_{s t} \widetilde{\mathcal{O}}_{t}^{(n)} .
$$

Since $\mathcal{O}_{t}^{(n)} \stackrel{d}{\rightarrow} \mathcal{O}_{t}^{L}$ and $\widetilde{\mathcal{O}}_{t}^{(n)} \stackrel{d}{\rightarrow} \mathcal{O}_{t}^{\tilde{L}}$, by the second part of Theorem 1 , we get

$$
\mathcal{O}_{t}^{L} \preceq_{s t} \mathcal{O}_{t}^{\tilde{L}} .
$$

This means that

$$
\mathbb{P}_{x}\left(\mathcal{O}_{t}^{L}>r\right) \leq \mathbb{P}_{x}\left(\mathcal{O}_{t}^{\tilde{L}}>r\right), \text { for all } r .
$$

The property in Equation (16) follows.

The monotonicity property in Equation (16) says that the risk measure $\rho_{\epsilon}^{(r, t)}[L]$ is increasing with respect to the stochastic dominance order. Note that, if $\mathbb{P}\left(L_{t} \leq \tilde{L}_{t}\right)=1$ for all $t \geq 0$, then we can also prove that

$$
\rho_{\epsilon}^{(r, t)}[L] \leq \rho_{\epsilon}^{(r, t)}[\tilde{L}]
$$


If we put together the monotonicity property in Equations (16) and (13), then we can deduce the following intuitive relationship: a smaller frequency and a smaller severity yield less occupation time in the red zone and thus a smaller probability of cumulative Parisian ruin. For example, by the third part of Theorem 1, if $C$ and $\tilde{C}$ are exponentially distributed random variables with parameters $\alpha$ and $\tilde{\alpha}$, respectively, and if $\alpha \geq \tilde{\alpha}, \lambda \leq \tilde{\lambda}$ and $c=\tilde{c}$, then, for a given common premium rate $c$, the initial capital needed at a given tolerance level $\epsilon$ is larger for $X$ than for $\tilde{X}$.

It is worth mentioning that, as an immediate consequence of Proposition 1, Theorem 2 is also satisfied for the infinite-time horizon risk measure $\zeta_{\epsilon}$. Thus, we have recovered some of the results in Properties 3.1 and 3.2 of Trufin et al. (2011). In addition, an important consequence of Proposition 1 is the stochastic ordering for the finite-time ruin probability $\psi(t, x)$.

When there is no initial reserve, i.e., when $x=0$, and for $c=\tilde{c}$, the last theorem generalizes Theorem 4 in Goovaerts and De Vylder (1984) and also Proposition 1 of Lefèvre et al. (2017).

\subsection{Relationship with Other Risk Measures}

Recall that our main object of study is the following VaR-type risk measure: for $r>0, \epsilon>0$ and $t>0$,

$$
\rho_{\epsilon}^{(r, t)}[L]=\inf \left\{x \geq 0: \psi_{r}(t, x) \leq \epsilon\right\}=\inf \left\{x \geq 0: \mathbb{P}_{x}\left(\mathcal{O}_{t}^{L}>r\right) \leq \epsilon\right\}
$$

When $t=\infty$, we write $\rho_{\epsilon}^{(r)}$.

We are also interested in the risk measure based on the finite-time probability of classical ruin:

$$
\zeta_{\epsilon}^{(t)}[L]=\inf \{x \geq 0: \psi(t, x) \leq \epsilon\}
$$

Using the inequality in Equation (9) and the discussions in the previous section, we deduce the following first proposition:

Proposition 1. For a given time horizon $0<t \leq \infty$ and an acceptance level $\epsilon>0$, the risk measure $\rho_{\epsilon}^{(r, t)}$ is less conservative than the risk measure $\zeta_{\epsilon}^{(t)}$, i.e.,

$$
\rho_{\epsilon}^{(r, t)}[L] \leq \zeta_{\epsilon}^{(t)}[L]
$$

and, when $r \rightarrow 0$, it converges to $\zeta_{\epsilon}^{(t)}$, i.e.,

$$
\rho_{\epsilon}^{(r, t)}[L] \uparrow \zeta_{\epsilon}^{(t)}[L], \text { as } r \rightarrow 0 .
$$

When the implementation delay $r$ is replaced by copies of an exponentially distributed random variable $\mathrm{e}_{q}$ with rate $q>0$, then, for $x \in \mathbb{R}$, we have

$$
\mathbb{P}_{x}\left(\sigma_{\mathrm{e}_{q}} \leq t\right)=1-\mathbb{E}_{x}\left[\mathrm{e}^{-q \mathcal{O}_{t}^{L}}\right] .
$$

In addition, in this case, cumulative Parisian ruin corresponds to Parisian ruin with exponential delays, that is

$$
\kappa^{q}=\inf \left\{t>0: t-g_{t}>\mathrm{e}_{q}^{g_{t}}\right\},
$$

where $g_{t}=\sup \left\{0 \leq s \leq t: X_{s} \geq 0\right\}$ is is the last time before $t$ when the process was non-negative.

Hence,

$$
\psi_{\mathrm{e}_{q}}(t, x)=\mathbb{P}_{x}\left(\sigma_{\mathrm{e}_{q}} \leq t\right)=\mathbb{P}_{x}\left(\kappa^{q} \leq t\right) .
$$

We can then define the following VaR-type risk measure: for $q, r>0, \epsilon>0$ and $t>0$,

$$
\rho_{\epsilon}^{(q, r, t)}[L]=\inf \left\{x \geq 0: \psi_{\mathrm{e}_{q}}(t, x) \leq \epsilon\right\}
$$


In addition, we have

$$
\rho_{\epsilon}^{(q, r, t)}[L] \uparrow \rho_{\epsilon}^{(r, t)}[L], \text { as } q \rightarrow \infty .
$$

The risk measure $\rho_{\epsilon}^{(q, r, t)}$ satisfies the properties in Theorem 2. For example, we proved that, if $L \preceq_{s t} \tilde{L}$, we have $\mathcal{O}_{t}^{L} \preceq_{s t} \mathcal{O}_{t}^{\tilde{L}}$. Then, using Equation (12), we obtain

$$
h\left(\mathcal{O}_{t}^{L}\right) \preceq_{s t} h\left(\mathcal{O}^{\tilde{L}}\right),
$$

where $h(x)=1-\mathrm{e}^{-q x}$. Hence, using Equation (11), we get

$$
\mathbb{E}_{x}[h(L)] \leq \mathbb{E}_{x}[h(\tilde{L})]
$$

and then

$$
\rho_{\epsilon}^{(q, r, t)}[L] \leq \rho_{\epsilon}^{(q, r, t)}[\tilde{L}]
$$

In addition, as an improvement of the finite-time version of the (infinite-horizon) risk measure defined by Loisel and Trufin (2014), we can define

$$
\omega_{a}^{(t)}[L]:=\inf \left\{x \geq 0: \mathbb{E}_{x}\left[\mathcal{A}_{t}^{L}\right] \leq a\right\}
$$

where $a>0$ is a tolerance level for the expected area in the red defined as

$$
\mathcal{A}_{t}^{L}=\int_{0}^{t}\left(L_{u}-x\right)_{+} \mathrm{d} u,
$$

where $(x)_{+}=\max (x, 0)$. Furthermore, we can use Theorem 1 of Loisel (2005) and then write

$$
\mathbb{E}_{x}\left[\mathcal{A}_{t}^{L}\right]=\int_{x}^{\infty} \mathbb{E}_{v}\left[\mathcal{O}_{t}^{L}\right] \mathrm{d} v=\int_{x}^{\infty} \int_{0}^{\infty} \mathbb{P}_{v}\left(\mathcal{O}_{t}^{L} \geq u\right) \mathrm{d} u \mathrm{~d} v
$$

Consequently, if we suppose that $L \preceq_{s t} \tilde{L}$, then $\mathcal{O}_{t}^{L} \preceq_{s t} \mathcal{O}_{t}^{\tilde{L}}$ and then, from Equations (10) and (24), we have

$$
\mathbb{E}_{x}\left[\mathcal{A}_{t}^{L}\right] \leq \mathbb{E}_{x}\left[\mathcal{A}_{t}^{\tilde{L}}\right]
$$

Thus,

$$
\omega_{a}^{(t)}[L] \leq \omega_{a}^{(t)}[\tilde{L}]
$$

which corresponds to Property 3.1 in Loisel and Trufin (2014).

Remark 1. Note that, with the distribution in Theorem 3, it is possible to compute the finite-time version of this risk measure based on the area in the red in the case of a Cramer-Lundberg process with exponential claims.

\section{Example: Cramér-Lundberg Model with Exponential Claims}

In this section, we want to see how $\rho_{\epsilon}^{(r, t)}$ reacts to changes in the value of its parameters. In other words, we want to perform a sensitivity analysis.

In general, we could use Monte Carlo simulations to compute values for $\rho_{\epsilon}^{(r, t)}$. However, if we consider a Cramér-Lundberg process with exponentially distributed claims $\left\{C_{1}, C_{2}, \ldots\right\}$ with rate parameter $\alpha>0$, then there exists an explicit expression for the distribution of the occupation time for a finite-time horizon. Unfortunately, such formulas are not available for most claim distributions.

Theorem 3 (Guérin and Renaud (2017)). For $t>0$, we have

$$
\mathbb{P}_{x}\left(\mathcal{O}_{t}^{L} \in \mathrm{d} s\right)=a_{t}^{x} \delta_{0}(\mathrm{~d} s)+\left(a_{t-s}^{x}+k_{t-s}^{x}\right)\left(\lambda-c \alpha\left(1-a_{s}^{0}\right)\right) \mathbf{1}_{(0, t)}(s) \mathrm{d} s,
$$


with

$$
a_{t}^{x}=1-\lambda \mathrm{e}^{-\alpha x} \int_{0}^{t} \mathrm{e}^{-(\lambda+c \alpha) s}\left[I_{0}(2 \sqrt{\lambda c \alpha s(s+x / c)})-\frac{s}{s+x / c} I_{2}(2 \sqrt{\lambda c \alpha s(s+x / c)})\right] \mathrm{d} s
$$

and

$$
k_{t}^{x}=\mathrm{e}^{-\alpha x}-1+\lambda x \alpha \mathrm{e}^{-\alpha x} \int_{0}^{t} \mathrm{e}^{-(\lambda+c \alpha) s}\left[I_{0}(2 \sqrt{\lambda c \alpha s(s+x / c)})-I_{2}(2 \sqrt{\lambda c \alpha s(s+x / c)})\right] \mathrm{d} s,
$$

where $I_{v}$ represents the modified Bessel function of the first kind of order $v$.

In Theorem $3, a_{t}^{x}$ is the survival ruin probability over $[0, t]$, that is

$$
\begin{aligned}
a_{t}^{x} & =1-\psi(t, x) \\
& =1-\lambda \mathrm{e}^{-\alpha x} \int_{0}^{t} \mathrm{e}^{-(\lambda+c \alpha) s}\left[I_{0}(2 \sqrt{\lambda c \alpha s(s+x / c)})-\frac{s}{s+x / c} I_{2}(2 \sqrt{\lambda c \alpha s(s+x / c)})\right] \mathrm{d} s .
\end{aligned}
$$

For an infinite-time horizon, we have the well-known expression:

$$
a^{x}=\lim _{t \rightarrow \infty} a_{t}^{x}=1-\psi(x)=\frac{\lambda}{c \alpha} \mathrm{e}^{x(\lambda / c-\alpha)}=\frac{1}{1+\eta} \mathrm{e}^{-x \alpha \eta /(1+\eta)} .
$$

From Corollary 2 in Renaud (2014), we can deduce the following expression for the distribution of $\mathcal{O}_{\infty}^{L}$, when the claims are exponentially distributed.

Corollary 1. For any $x \in \mathbb{R}$, we have

$$
\begin{aligned}
& \mathbb{P}_{x}\left(\mathcal{O}_{\infty}^{L} \in \mathrm{d} s\right)=a^{x} \delta_{0}(\mathrm{~d} s) \\
& +\frac{\lambda}{c}\left(1-\frac{\lambda}{c \alpha}\right) \mathrm{e}^{-c s \alpha} \mathrm{e}^{-x(\alpha-\lambda / c)}\left(c+\sum_{i=0}^{\infty} \frac{(\lambda s)^{i+1}}{i !(1+i) !}\left(c \Gamma(i+1, s \lambda)-\frac{c}{s \lambda} \Gamma(i+2, s \lambda)\right)\right),
\end{aligned}
$$

where $\Gamma(a, x)=\int_{0}^{x} \mathrm{e}^{-t} t^{a-1} \mathrm{~d} t$ is the incomplete gamma function.

The explicit formula in Theorem 3 allows for a sensitivity analysis of the value of the probability of cumulative Parisian ruin, when claims are exponentially distributed, with respect to the delay parameter $r$ and the time horizon $t$. In Figure 2, we observe that for a fixed delay parameter $r$, the probability of cumulative Parisian ruin increases when the time horizon $t$ increases. This is because we accumulate more occupation time. On the other hand, it decreases when the delay $r$ increases. For a fixed value of the time horizon $t$, increasing the initial capital $x$ decreases the probability of cumulative Parisian ruin, as expected.

For the corresponding risk measures, Figure 3 illustrates the relationships in Equations (19) and in (20) between $\rho_{\epsilon}^{(r, t)}$ and $\zeta_{\epsilon}^{(t)}$. As $r \rightarrow 0$, i.e., as the grace period gets smaller, the initial capital needed with $\rho_{\epsilon}^{(r, t)}$ increases toward that needed with $\zeta_{\epsilon}^{(t)}$, both at a tolerance level of $\epsilon=0.3$. When the time horizon $t$ increases, both risk measures increase the initial capital needed for that tolerance level. 

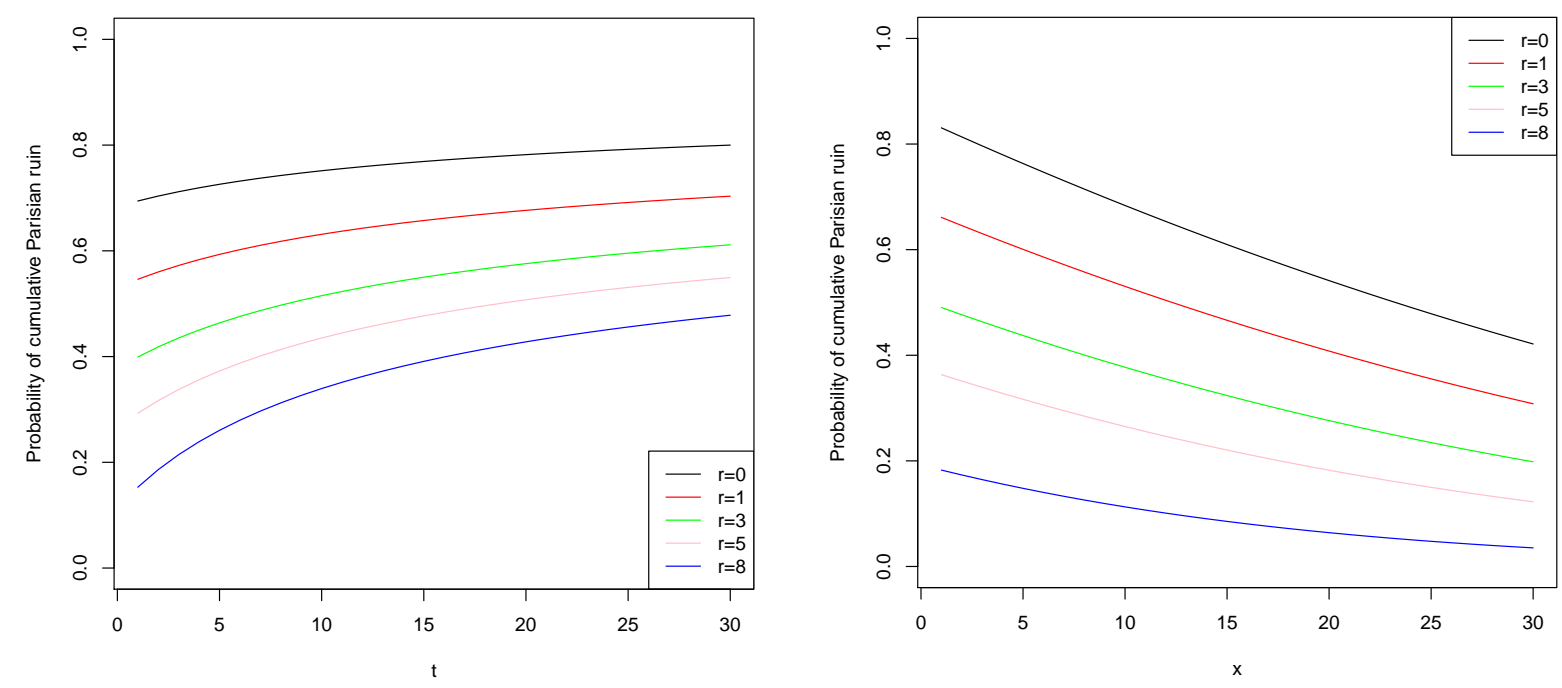

Figure 2. The probability of cumulative Parisian ruin for the Cramér-Lundberg process with $\alpha=1 / 8$, $\lambda=2, c=17, r=1, x=10$ and $t=10$.
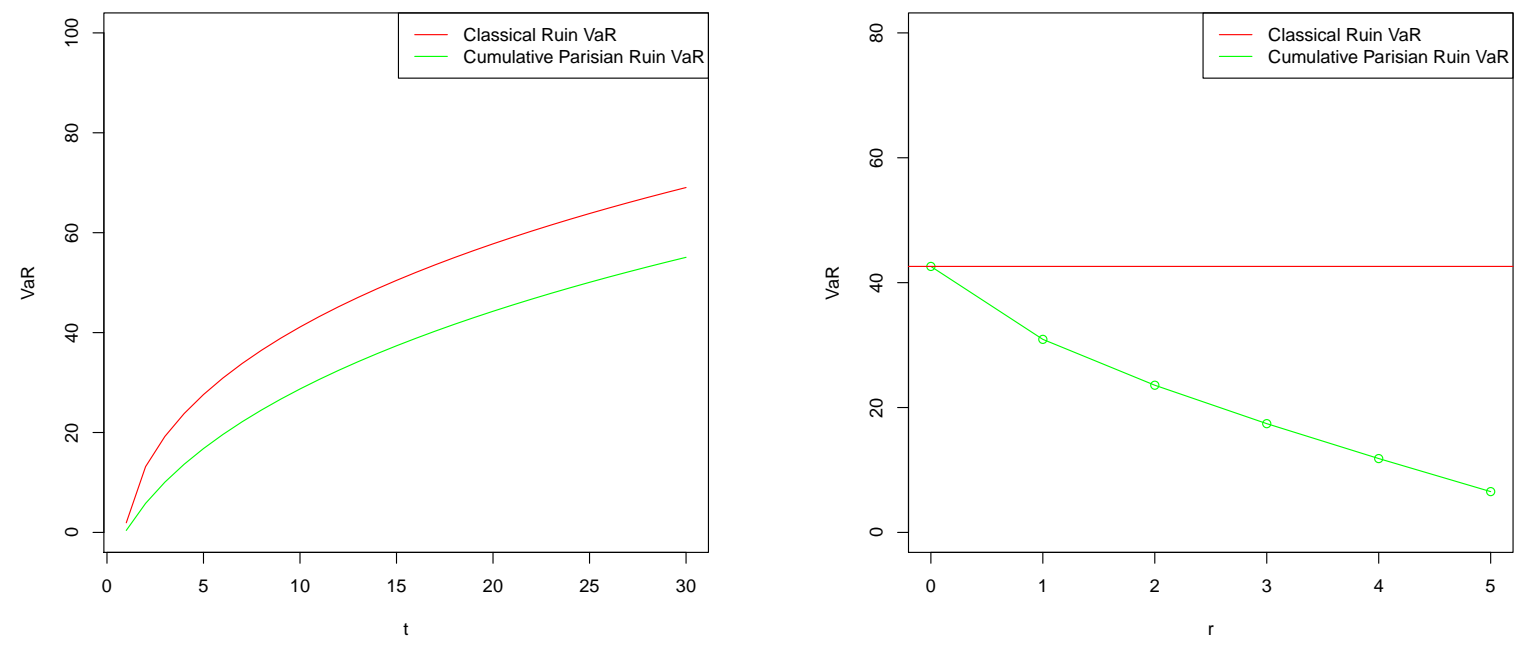

Figure 3. Risk measures $\rho_{\epsilon}^{(r, t)}$ and $\zeta_{\epsilon}^{(t)}$ for the Cramér-Lundberg process with $\alpha=1 / 8, \lambda=2, c=17$, $t=10, r=2$, and $\epsilon=0.3$.

\section{Conclusions}

In this paper, we study a VaR-type risk measure derived from cumulative Parisian ruin for the Cramér-Lundberg risk process. Precisely, this measure is defined as the smallest amount of capital for which the associated cumulative Parisian ruin probability is less than or equal to a tolerable level. We derive some properties of this risk measure and we provide some relationships with other risk measures. Finally, for exponentially distributed claims sizes, we performed sensitivity analysis of the values of the probability of cumulative Parisian ruin and the risk measure. Our risk measure could still be used for other risk processes such as the Brownian motion risk model.

Author Contributions: All the authors collaborated equally at every step of the research and in writing the paper.

Acknowledgments: Funding in support of this work was provided by the Natural Sciences and Engineering Research Council of Canada (NSERC). M.A.L. thanks the Institut des sciences mathématiques (ISM) and the Faculté des sciences at UQAM for their doctoral scholarships.

Conflicts of Interest: The authors declare no conflict of interest. 


\section{References}

Beekman, John A. 1985. A series for infinite time ruin probabilities. Insurance: Mathematics and Economics 4: 129-34. [CrossRef]

Cramér, Harald. 1930. On the Mathematical Theory of Risk. Alingsås: Centraltryckeriet.

Guérin, Hélène, and Jean-François Renaud. 2017. On the distribution of cumulative Parisian ruin. Insurance: Mathematics and Economics 73: 116-23. [CrossRef]

Goovaerts, Marc, and F. Etienne De Vylder. 1984. Dangerous Distributions and Ruin Probabilities in the Classical Risk Model. Paper presented at the 22nd International Congress of Actuaries, Sydney, Australia, October 21-27; pp. 111-20.

Denuit, Michel, Jan Dhaene, Marc Goovaerts, and Rob Kaas. 2005. Actuarial Theory for Dependent Risks: Measures, Orders and Models. New York: Wiley.

Shaked, Moshe, and George Shanthikumar. 2007. Stochastic Orders. New York: Springer.

Kaas, Rob, Marc Goovaerts, Jan Dhaene, and Michel Denuit. 2008. Modern Actuarial Risk Theory Using R. Berlin/Heidelberg: Springer.

Loisel, Stéphane. 2005. Differentiation of some functionals of risk processes, and optimal reserve allocation. Journal of Applied Probability 42: 379-92. [CrossRef]

Loisel, Stéphane, and Julien Trufin. 2014. Properties of a risk measure derived from the expected area in red. Insurance: Mathematics and Economics 55: 191-9. [CrossRef]

Lefèvre, Claude, Julien Trufin, and Pierre Zuyderhoff. 2017. Some comparison results for finite-time ruin probabilities in the classical risk model. Insurance: Mathematics and Economics 77: 143-49. [CrossRef]

Lundberg, Filip. 1903. I. Approximerad framställning af sannolikhetsfunktionen. II. Återförsäkring af kollektivrisker. Akademisk afhandling, etc. Uppsala: Almqvist \& Wiksell.

Renaud, Jean-François. 2014. On the time spent in the red by a refracted Lévy risk process. Journal of Applied Probability 51: 1171-88. [CrossRef]

Mitric, Ilie-Radu, and Julien Trufin. 2016. On a risk measure inspired from the ruin probability and the expected deficit at ruin. Scandinavian Actuarial Journal 2016: 932-51. [CrossRef]

Trufin, Julien, Hansjoerg Albrecher, and Michel Denuit. 2011. Properties of a risk measure derived from ruin theory. The Geneva Risk and Insurance Review 36: 174-88. [CrossRef]

(C) 2018 by the authors. Licensee MDPI, Basel, Switzerland. This article is an open access article distributed under the terms and conditions of the Creative Commons Attribution (CC BY) license (http://creativecommons.org/licenses/by/4.0/). 